\title{
The Neuronal Basis of the Behavioral Choice between Swimming and Shortening in the Leech: Control Is Not Selectively Exercised at Higher Circuit Levels
}

\author{
Brian K. Shaw and William B. Kristan Jr. \\ Department of Biology, University of California San Diego, La Jolla, California 92093-0357
}

\begin{abstract}
Swimming and the whole-body shortening reflex are two incompatible behaviors performed by the medicinal leech Hirudo medicinalis. We set out to examine the neuronal basis of the choice between these behaviors, taking advantage of the fact that the neuronal circuit underlying swimming is relatively well understood. The leech swim circuit is organized hierarchically and contains three interneuronal levels, including two upper levels of "command-like" neurons. We tested the responses of the swim circuit neurons to stimuli that produced shortening, using reduced preparations in which neurophysiological recording could be performed while behaviors were elicited. We found that the majority of the swim circuit neurons, including most of the command-like cells and all of the cells at the
\end{abstract}

highest hierarchical level of the circuit, were excited by stimuli that produced shortening as well as by stimuli that produced swimming. Only a subset of neurons, at levels below the top, were inhibited during shortening; these included one of the command-like cells and an oscillator cell (an interneuron that is part of the central pattern generator for swimming). These results imply that the control of the choice between swimming and shortening is not exercised selectively at the higher levels of the swim circuit.

Key words: Hirudo medicinalis; swimming; shortening; behavioral choice; decision-making; motor control; command neuron
The neuronal mechanisms that underlie behavioral decisionmaking are little understood. One long-standing proposal is that behavior-dedicated "command" neurons trigger particular behaviors and that inhibitory interactions between such higher-order neurons allow for choices between behaviors (Kupfermann and Weiss, 1978; Kovac and Davis, 1980; Edwards, 1991). To date, however, only a few identified "command-like" neurons have been examined to see whether in fact they do act as behavior-dedicated decision points for choices between behaviors (Krasne and Lee, 1988; Huang and Satterlie, 1990; Jing and Gillette, 1995; Norekian and Satterlie, 1996); for these cases at least, the answer seems to be positive. To test this further, we examined the behavioral choice between swimming and shortening in the medicinal leech Hirudo medicinalis, taking advantage of the fact that the neuronal circuit underlying swimming has been studied extensively and is relatively well understood (for review, see Friesen, 1989a; Brodfuehrer et al., 1995a). This circuit is particularly well suited to the investigation of this issue, because a comparatively large and diverse group of higher-order command-like neurons have been identified.

The swim circuit is organized hierarchically and contains three interneuronal levels. The hierarchical nature of the circuit is apparent both in the functional properties of the neurons and in

Received Aug. 9, 1996; revised Oct. 30, 1996; accepted Oct. 31, 1996.

This work was supported by a National Science Foundation predoctoral fellowship (B.K.S.), National Institutes of Health Training Grant GM08107 (B.K.S.), and National Institute of Mental Health Research Grant MH43396 (W.B.K.). We thank J. Lewis, C. Hempel, A. Selverston, P. Brodfuehrer, and a number of anonymous reviewers for helpful criticism of various versions of this manuscript. B.K.S. thanks W. O. Friesen for training in identifying oscillator cells and P. Brodfuehrer for advice regarding the identification of cell SE1.

Correspondence should be addressed to Brian K. Shaw, Department of Biology 0357, University of California San Diego, La Jolla, CA 92093-0357.

Copyright (C) 1997 Society for Neuroscience $0270-6474 / 97 / 170786-10 \$ 05.00 / 0$ the direction of synaptic flow, which is largely from top down (Fig. $1 A$ ). (For a detailed exposition of the evidence for this hierarchical model, see Friesen, 1989a.) The neurons in the uppermost two levels of the circuit, the swim-activating neurons of the head brain and the segmental swim-gating neurons, share the command-like property of being sufficient to initiate swimming behavior when they are excited by intracellular current injection (Weeks and Kristan, 1978; Brodfuehrer and Friesen, 1986a; Nusbaum and Kristan, 1986; Brodfuehrer et al., 1995b). Here we will refer to these neurons as command-like cells, because none of them has been shown to be individually necessary for swimming, which is a widely accepted condition for classifying a cell as a true command neuron (Kupfermann and Weiss, 1978). It has been believed that the command-like cells of the swim circuit are in fact part of the normal pathway for the decision to swim, because stimuli that naturally cause swimming, such as mechanical stimulation of the posterior region (Kristan et al., 1982), excite these cells before the onset of swimming (Weeks and Kristan, 1978; Brodfuehrer and Friesen, 1986b; Nusbaum and Kristan, 1986) (Fig. 1B).

Although a mechanical stimulus delivered to posterior regions of a leech tends to cause it to swim, a mechanical stimulus to anterior regions tends to cause a very different response: a wholebody shortening reflex, which consists of a rapid and nearly synchronous contraction of the body (Kristan et al., 1982) (Fig. $2 A$ ). Recently we have worked out a method to reliably obtain the shortening reflex (and/or its motor pattern) in reduced preparations, which has allowed us to characterize some of the neuronal underpinnings of this behavior (Shaw and Kristan, 1995a). In the present study we examined the neuronal basis of the behavioral choice between swimming and shortening by testing the response of swim circuit neurons to stimuli that produced shortening. Our aim was to determine whether the control of the choice between swimming and shortening is exercised at the higher levels of the 

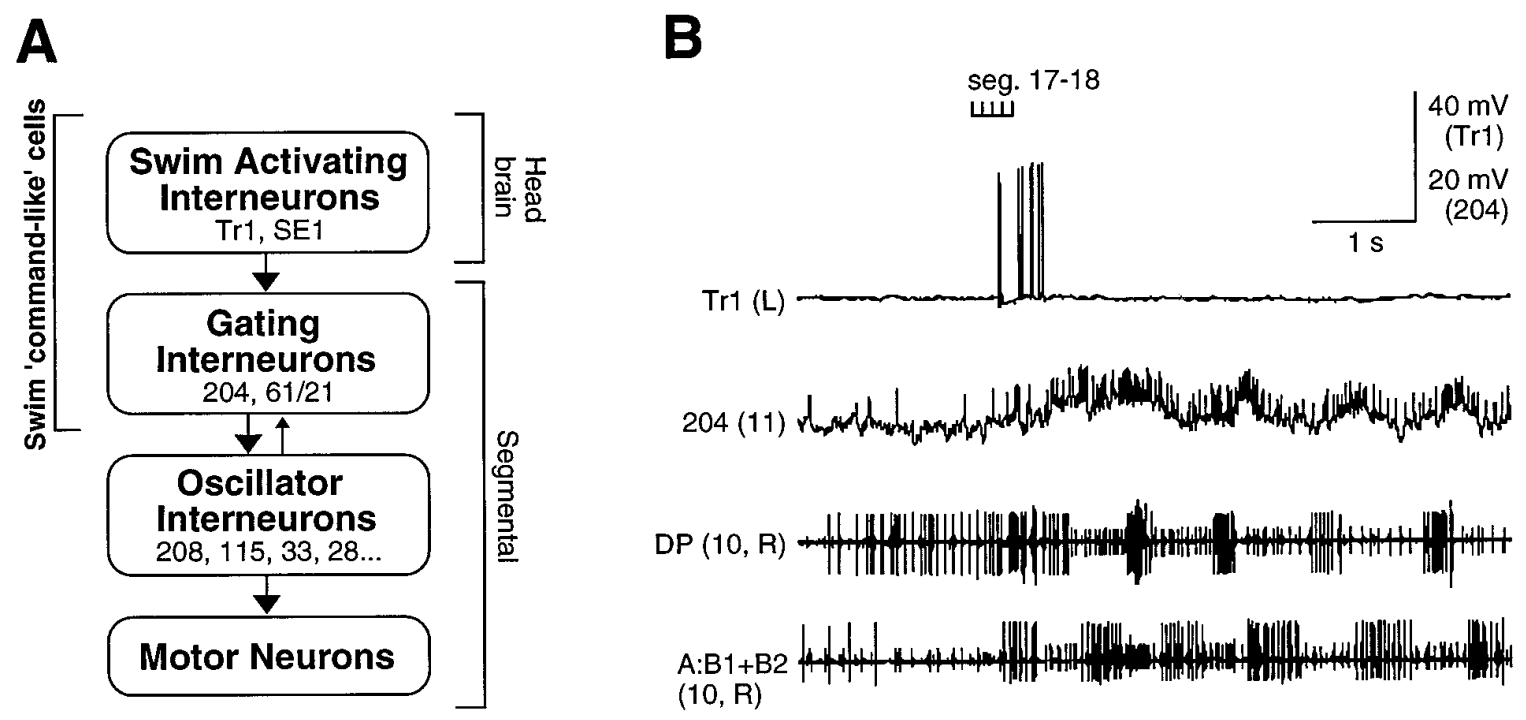

Figure 1. The neuronal basis of swimming in the leech (Friesen, 1989a; Brodfuehrer et al., 1995a). A, Schematic diagram of the swim circuit. Identified neurons from each functional level are given in the boxes. The synaptic flow is mostly top down: the swim-activating neurons excite the gating neurons, which excite the oscillator neurons, which in turn drive the motor neurons. This is denoted schematically by the arrows. There is some feedback from the oscillator to the gating level, indicated by the smaller arrow. Interneurons are assigned to a given level on the basis of their functional properties as well as their synaptic inputs and outputs. The top-level neurons, trigger cell $1(\operatorname{Tr} 1)$ and swim excitor 1 (SE1), can initiate prolonged swim episodes when briefly depolarized. The gating neurons, at the next level down, require sustained current injection to initiate and maintain a swim episode. At the lowest interneuronal level are the oscillator neurons, which do not initiate swimming when depolarized; these cells make up the central pattern generator for the behavior. Although cell SE1 has the "trigger" property of being able to elicit swimming when briefly activated, it has been hypothesized that this cell normally functions as a "gain-control" for swim-initiating inputs rather than as a trigger (Brodfuehrer et al., 1995b). Trigger cell 2 (Tr2) is not included in the swim-activating category because a recent study has shown that this cell is more effective at terminating swimming than initiating it (O'Gara and Friesen, 1995). B, A swim-initiating stimulus excited cells $\operatorname{Tr} 1$ and 204 before the onset of swimming. The stimulus (indicated by the horizontal bar, with individual pulses indicated by the vertical tics; see Materials and Methods) was delivered to the posterior dorsal skin of a semi-intact preparation, between segments 17 and 18 (seg. 17-18). The activation of cell Tr1 is brief, but cell 204 remains active for the duration of the swim episode, receiving some cyclic feedback from the oscillator level. The stimulus caused cell $\operatorname{Tr} 1$ to fire eight spikes. The motor pattern for swimming is characterized by alternating bursts in dorsal excitor (DE) and ventral excitor (VE) motor neurons, which can be seen in the extracellular recordings (see Materials and Methods). This preparation had an intact posterior portion, with the nerve cord exposed from the head brain to segment 15 . It was bathed in saline containing $50 \mu \mathrm{M}$ serotonin to lower the threshold for swimming (Willard, 1981). In this and subsequent figures, the numbers in parentheses in the trace labels indicate from which segmental ganglion the recording was made.

swim circuit, as predicted by the proposed decision-making strategy described above. We examined a number of questions in this regard. Are the swim command-like cells inhibited by stimuli that produce shortening? In particular, are the cells at the top level of the circuit inhibited? And, finally, is inhibition selectively targeted only to command-like cells?

Parts of this paper have been published previously in abstract form (Shaw and Kristan, 1993; Shaw and Kristan, 1995b).

\section{MATERIALS AND METHODS}

Preparation and procedure. Adult $H$. medicinalis were obtained from Leeches USA (Westbury, NY). The general methods and experimental procedure were as described previously (Kristan et al., 1974; Shaw and Kristan, 1995a). Physiological preparations were used in which the responses of neurons could be monitored while behaviors, or the neural correlates of behaviors, were elicited. The leech nervous system consists of a head brain, a tail brain, and 21 segmental ganglia, linked by connectives into a nerve cord. The types of preparation used in most of the experiments are diagrammed in Figure $2 B$. Most recordings from segmental neurons were performed on semi-intact preparations with an anterior portion intact (from the head to segment 7-9) and the nerve cord exposed for recording posterior to this. Recordings from head-brain neurons were made in more reduced preparations in which only a ring of body wall was left attached to the nerve cord, from segments $4-6$, and the cord was exposed anterior and posterior to this. In the standard semiintact preparations, the head and tail brains were generally removed to reduce response variability and promote reliable swimming (Kristan et al., 1982; Brodfuehrer and Friesen, 1986c); in the more reduced preparations, only the tail brain was removed.

The whole-body shortening reflex (and/or its characteristic motor pat- tern) was elicited in these preparations using stimulating electrodes implanted in the dorsal anterior skin, between segments 3 and 4 or segments 4 and 5 . The electrodes and the method of implanting them were as described in Shaw and Kristan (1995a). The stimulus was a 0.5 sec, $10 \mathrm{~Hz}$ train of shocks ( $1 \mathrm{msec}$ pulses, $8 \mathrm{~V}$ intensity), which mimics a mechanical stimulus (Kristan et al., 1982; Shaw and Kristan, 1995a). Intervals of $\geq 3$ min were allowed between trials. Swimming was elicited in these preparations with trains of shocks delivered to posterior nerves via a suction electrode.

In a few experiments, semi-intact preparations with an intact posterior portion, and with stimulating electrodes implanted in the posterior skin rather than the anterior, were used to investigate the initiation of swimming (e.g., Fig. $1 B$ ). The stimulus protocol was the same as for anterior stimuli.

Monitoring of behavior. In a subset of the semi-intact preparations, shortening behavior was monitored with a tension transducer attached to the front end of the animal, as described in Shaw and Kristan (1995a). The line that connected the animal to the transducer was set with some slack at rest, so that the animal could swim unimpeded; only during whole-body shortening was the transducer engaged.

Physiology. Intracellular recordings were made with 30-40 M $\Omega$ electrodes filled with $3 \mathrm{M} \mathrm{KAc}$. Extracellular recordings were made with suction electrodes. To facilitate the penetration of cells, the connective sheath surrounding the segmental ganglion or head brain to be recorded from was usually removed with fine scissors. Most cells were identified by their soma position and their physiological properties; the properties used for identification included spike appearance, synaptic interactions, motor effects, and/or the activity pattern of the cell during swimming (Friesen et al., 1978; Weeks and Kristan, 1978; Weeks, 1982b; Brodfuehrer and Friesen, 1986a; Friesen, 1989b; Brodfuehrer et al., 1995b). Cells 61 and 21, which contain serotonin, were identified by brief staining with $0.0005 \%$ Neutral Red (Nusbaum and Kristan, 1986). 
A

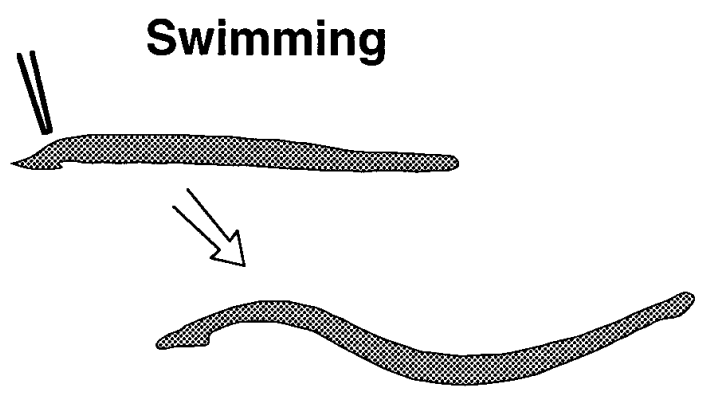

POSTERIOR

ANTERIOR

\section{Shortening}

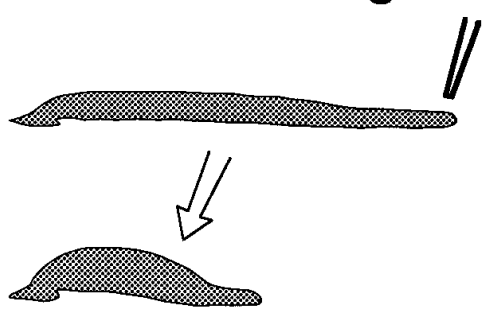

B

\section{Semi-intact preparation}

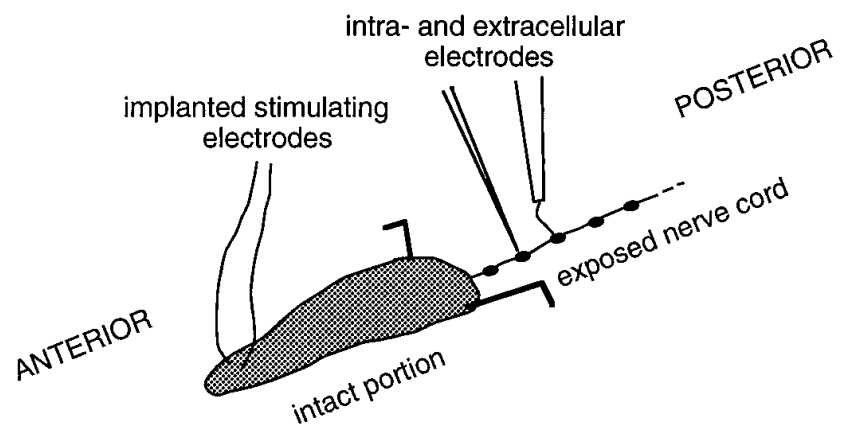

\section{Reduced semi-intact preparation with the head brain exposed}

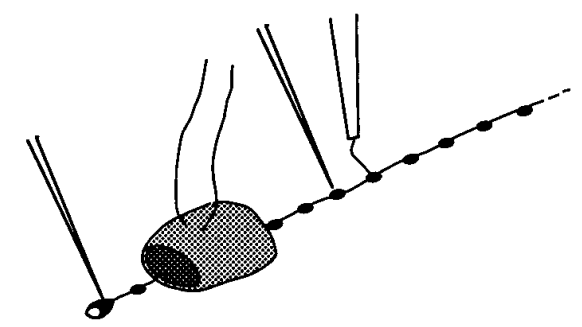

Figure 2. Schematic drawings of the behaviors studied and the physiological preparations used. $A$, Representations of the swimming and shortening behaviors. Posterior mechanical stimuli tend to cause swimming, whereas anterior stimuli tend to cause shortening (Kristan et al., 1982) Swimming consists of waves of dorsal-ventral undulations that run from the front to the back of the animal. The whole-body shortening reflex
In some cases the stimulus caused movement of the nerve cord itself; the result of this was that a portion of the intracellular recordings (a minority) contained movement artifacts. The stimulus could cause movement of the nerve cord because the cord contains muscle fibers (Tulsi and Coggeshall, 1971; Magni and Pellegrino, 1978). Movement artifacts tended to be more of a problem in standard semi-intact preparations but could also occur in more reduced preparations. When such artifacts were seen, they occurred at latencies $\geq 150 \mathrm{msec}$ from stimulus onset, which is greater than the latencies for postsynaptic potentials (PSPs) in swim circuit neurons (see Table 1); thus the qualitative nature of a response could usually be determined even if an artifact occurred. The occurrence of a movement artifact, however, usually prevented computation of the peak PSP magnitude (see below) and spike histogram for that trial (e.g., Fig. 6B).

Depending on the experiment, extracellular recordings were made from the dorsal posterior (DP) nerve or its branches, the first two branches of the anterior root $(\mathrm{A}: \mathrm{B} 1+\mathrm{B} 2)$, and the connective between two ganglia. DP and its second branch (DP:B2) contain a large spike from cell 3, a dorsal excitor (DE) motor neuron (Ort et al., 1974; Shaw and Kristan, 1995a). DP (but not DP:B2) also contains a large spike from the $\mathrm{L}$ cell, a motor neuron that excites both dorsal and ventral muscles (Ort et al., 1974). A:B1+B2 contains the spikes of ventral excitor (VE) motor neurons (Ort et al., 1974) (B.K.S., personal observation). The largest spike in the connective is that of the S cell (Bagnoli et al., 1972), an interneuron that along with its segmental homologs forms an electrically coupled chain that runs the length of the nerve cord.

The motor pattern for shortening is characterized by short-latency coactivation of the DE, the VE, and the L cell; at an interneuronal level, shortening involves the activation of the $\mathrm{S}$ cell network and other fast interneuronal pathways in the connective (Shaw and Kristan, 1995a). For all of the data presented in this paper on the responses of swim circuit neurons during shortening, simultaneous extracellular recordings were made at least from the DP nerve (or its branches) and the connective to ensure that the shortening motor pattern was expressed when the stimulus was given.

Physiological data were recorded and displayed as described in Shaw and Kristan (1995a).

Data analysis. The peak magnitudes of the PSPs produced by the shortening stimulus were calculated as follows. First, traces were lowpass-filtered (50 Hz cutoff) to remove stimulus artifacts and attenuate spikes. The baseline was defined as the mean membrane potential for the $0.5 \mathrm{sec}$ before the onset of the stimulus, and the peak PSP was defined as the deviation from that baseline with the greatest absolute value during the $1 \mathrm{sec}$ after stimulus onset.

\section{RESULTS}

Swimming and shortening are incompatible behaviors

Swimming and shortening use most of the same motor neurons, but in different patterns (Shaw and Kristan, 1995a). This is exemplified in Figure $3 A$, in which the activities of the same motor neurons are shown during the performance of both behaviors. The implication of this is that the two behaviors are incompatible and cannot be coexpressed. This can be demonstrated directly by putting the behaviors into conflict. In Figure $3 B$, a "shortening" stimulus was delivered to a swimming leech, causing a behavioral switch from swimming to shortening. No mix or blend between the behaviors was observed, but rather a sharp transition from one to the other. Such switches from swimming to shortening have been seen reliably in eight semi-intact preparations. This result implies that there must exist mechanisms to prevent the simultaneous expression of both motor patterns, i.e., there must be a choice between the behaviors. The result also suggests that shortening dominates swimming in the "behavioral hierarchy" of the leech.

consists of a rapid, nearly synchronous contraction of all or most of the body, which causes the head to be retracted away from the stimulus. $B$, Representations of the physiological preparations that were used for most of the experiments (see Materials and Methods). 
A

$3(11, L)$

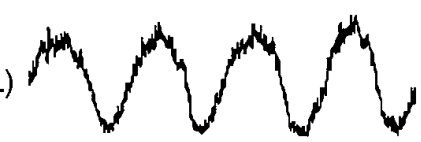

$\mathrm{DP}: \mathrm{B} 2$

$(11, \mathrm{R})$
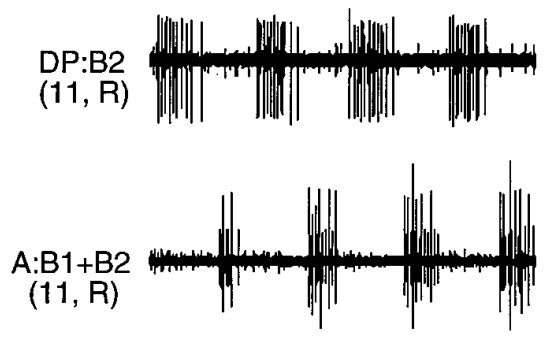

Shortening

seg. 3-4

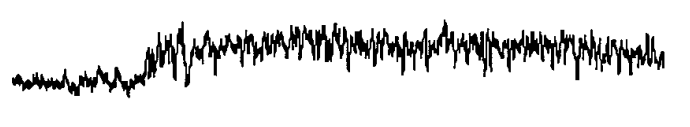

$20 \mathrm{mV}$

$1 \mathrm{~s}$
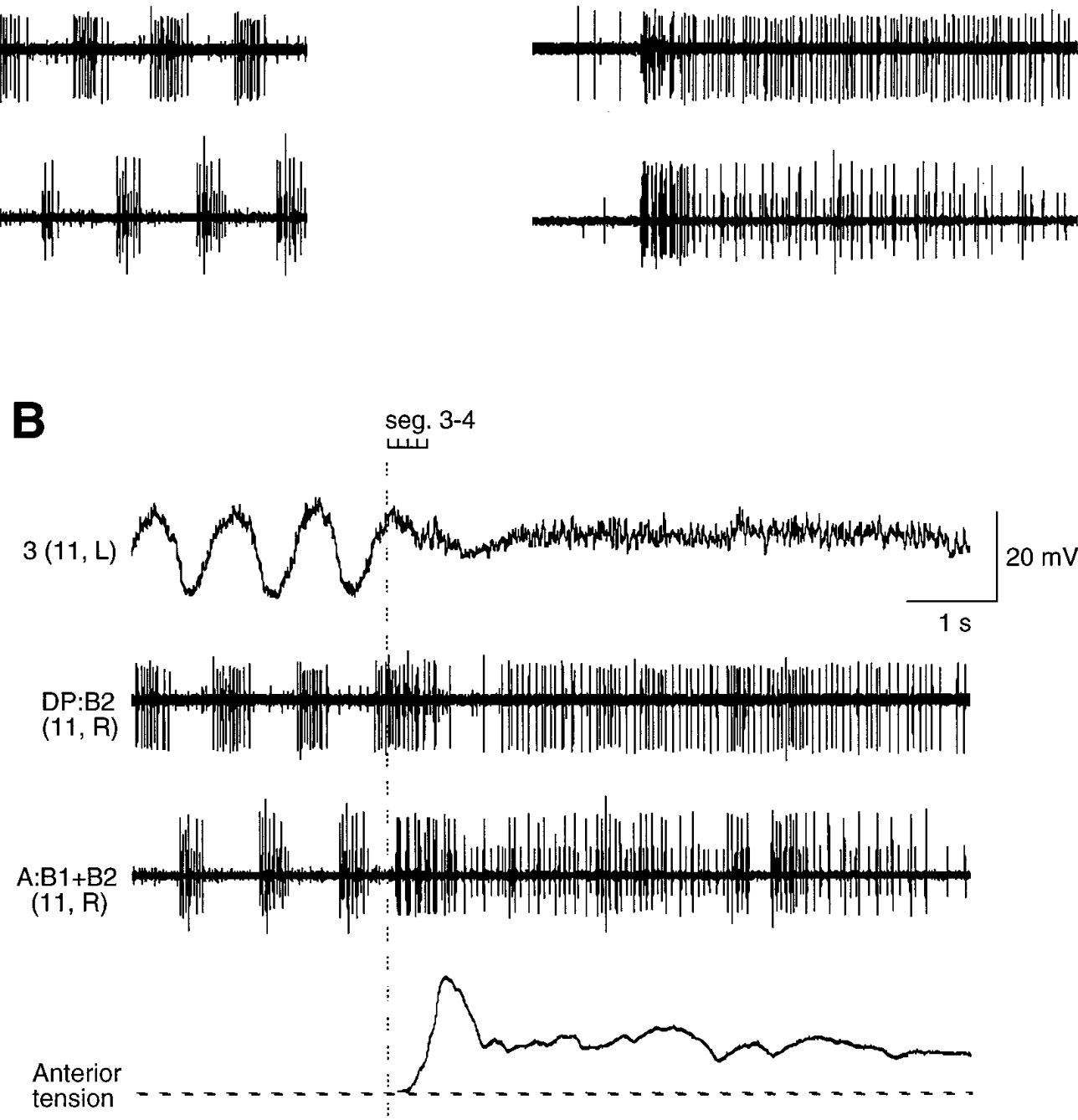

Figure 3. The motor patterns and interaction of swimming and shortening. $A$, Recordings taken during swimming and shortening in the same semi-intact preparation. Cell 3 is a DE motor neuron that accounts for the large spike in the DP:B2 recording. Spikes recorded intracellularly in the soma of cell 3 are relatively small. During swimming, the DE and VE fired in alternating bursts. In contrast, during shortening the DE and VE were coactivated. In this and subsequent figures, the stimulus for shortening is indicated by the horizontal bar (with individual stimulus pulses indicated by the vertical tics), and the location of the stimulating electrodes is given just over the bars. $B, \mathrm{~A}$ "shortening" stimulus delivered to a swimming leech caused a behavioral switch from swimming to shortening. This can be seen at the motor neuron level in the transition from alternation to coactivation in the DE and VE, and at the behavioral level in the sharp rise in anterior tension. These recordings were made in the same semi-intact preparation as $A$. The dotted baseline of the tension trace is meant to indicate that the transducer is somewhat slack at rest (see Materials and Methods); because of this, no magnitude scale is shown for the tension recording.

\section{Responses of swim circuit neurons during shortening}

We began our survey of the swim circuit with cell 204, a gating neuron that has particularly powerful swim-initiating effects. Cell 204 was strongly inhibited by stimuli that caused shortening (Fig. 4). The inhibition had a short latency, occurring at approximately the same time that the shortening motor pattern was expressed. This first result, at least, was in accord with the prediction that the swim command-like cells would be inhibited during shortening.
As we expanded the survey, however, the results grew more complicated.

The swim-activating neurons, at the highest level of the swim circuit, were tested in reduced semi-intact preparations with the head brain exposed. Both of these neurons, trigger cell 1 (Tr1) and swim excitor 1 (SE1), were excited by stimuli that caused shortening (Fig. 5). An EPSP could be seen in the soma of cell $\mathrm{SE} 1$, but for cell $\mathrm{Tr} 1$ the spikes rose directly from the resting 

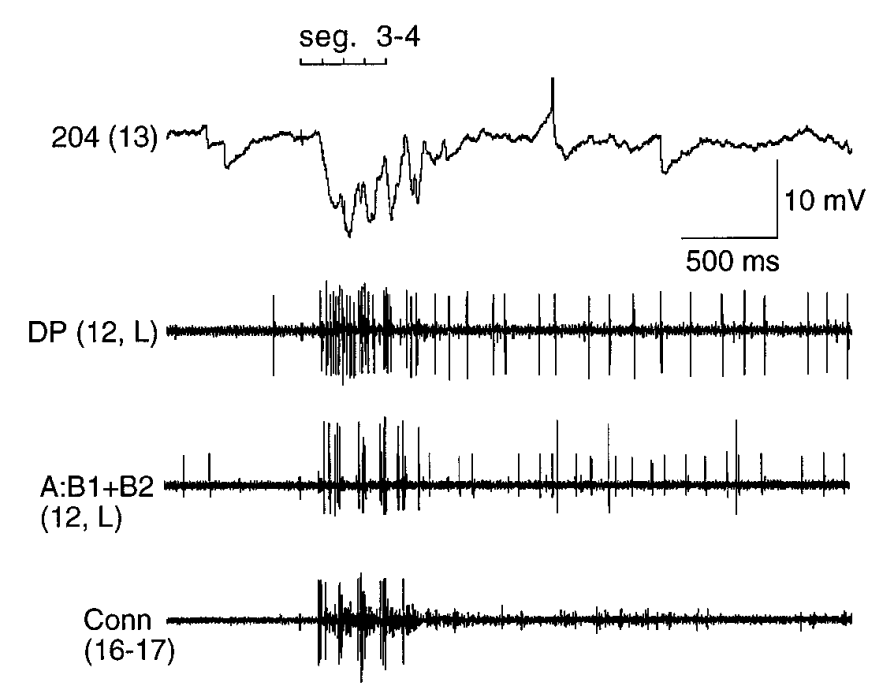

Figure 4. The response of cell 204 during shortening in a semi-intact preparation. The motor pattern for shortening, coactivation of the DE, the $\mathrm{VE}$, and the $\mathrm{L}$ cell, can be seen in the extracellular recordings from nerves. The activation of the $\mathrm{S}$ cell network and other interneuronal pathways can be seen in the extracellular recording from the connective. (See Materials and Methods.)

potential. This is presumably because the synaptic contacts from sensory neurons onto cell $\operatorname{Tr} 1$ are electrotonically distant from the soma. The excitation of these cells occurred at the same time that cell 204 was inhibited. This finding-that the highest-level neurons of the swim circuit, which can initiate swimming when stimulated, were excited by stimuli that produce shortening-was surprising. For the case of cell $\operatorname{Tr} 1$, however, the result is consistent with the report that cell $\operatorname{Tr} 1$ receives mechanosensory input from the anterior of the animal as well as more posterior regions (Brodfuehrer and Friesen, 1986b). The degree to which the swimactivating neurons were excited during shortening (Fig. 5B) is considerably below the levels of excitation required for them to initiate swimming when stimulated intracellularly: the firing frequencies necessary to elicit swimming are $30-50 \mathrm{~Hz}$ for cell $\operatorname{Tr} 1$ (Brodfuehrer and Friesen, 1986a) and 50-70 Hz for cell SE1 (Brodfuehrer et al., 1995b); however, mechanical stimuli that cause swimming do not excite the swim-activating cells to these levels either (Brodfuehrer and Friesen, 1986b; Brodfuehrer et al., 1995b). In fact, for the case of cell $\operatorname{Tr} 1$, the amount of excitation produced by stimuli that caused swimming and stimuli that caused shortening was similar (compare Figs. 1, 5).

At the next level down from the swim-activating neurons are the gating neurons, which were tested for the most part in semi-intact preparations. As reported earlier, cell 204 was inhibited (Fig. 6). The other gating neurons, however, showed the opposite effect. Cell 61 was excited during shortening (Fig. 6). Cell 21, which is functionally very similar to cell 61 (Nusbaum and Kristan, 1986), showed an excitatory response similar to that of cell 61 (data not shown). These results indicate that even at the same level of the swim circuit there can be opposite effects on different cells. They also demonstrate, in combination with the results from the swimactivating neurons, that only one of the five identified commandlike neurons of the swim circuit was inhibited by stimuli that produce shortening; the others were excited by such stimuli.

The cells of the lowest interneuronal level, the oscillator neurons, were tested in semi-intact preparations. As at the gating level, different oscillator cells showed opposite effects. Cell 208 was inhibited during shortening, in a manner quite similar in timing and appearance to the inhibition of cell 204 (Fig. 6). Cell 115, in contrast, was excited (Fig. 6). That cell 208 is inhibited implies that, just as not all the command-like cells are inhibited during shortening, not all the cells that show inhibition during shortening are command-like. Thus, inhibitory signals are not selectively targeted only to command-like neurons. Other oscillator cells were also tested. Cell 33 was inhibited, more weakly and with longer latency than cells 204 and 208; cell 28 was excited (data not shown).

Anterior stimuli produced shortening motor responses in both standard semi-intact preparations and the more reduced semiintact preparations with the head brain exposed; however, we found that the motor responses in the more reduced preparations tended to be weaker. This is consistent with our earlier observation that more extensive dissection weakens the shortening response (Shaw and Kristan, 1995a). In accordance with this, the responses of cell 204, although inhibitory in both cases, were smaller in the more reduced preparations (compare Figs. 5, 6). Although this does not affect our conclusions regarding the qualitative pattern of responses, it does create a complication for any quantitative comparisons between the two different types of preparation.

\section{Response latencies of swim circuit neurons}

The response latencies of swim circuit neurons to shortening stimuli are shown in Table 1 . These latencies, which reflect the time it takes for signals to travel from the anterior to the midbody of the leech, are quite short; they are comparable to the response latencies of midbody motor neurons during shortening (Shaw and Kristan, 1995a). This suggests that some of the same interneuronal pathways that drive motor neurons during shortening could also be responsible for inhibiting or exciting swim circuit interneurons.

The inhibition of cells 204 and 208 appears quite similar in approximate time course (Fig. $6 A$ ), suggesting that these two cells might share common inputs; however, cell 208 tended to have a slightly shorter initial response latency than cell 204 (Table 1). Cells 204 and 208 were recorded simultaneously in the same ganglion in three preparations. In two of these preparations, the cell 208 IPSP preceded that of cell 204 (by $\sim 13 \mathrm{msec}$ ), but in the third, their latencies were similar. These results warrant no strong

\begin{tabular}{lllll}
\hline \multicolumn{5}{l}{ Table 1. Response latencies of swim circuit neurons } \\
\multicolumn{6}{c}{ Cell } & Ganglion & $\begin{array}{l}\text { Mean } \\
(\mathrm{msec})\end{array}$ & $\begin{array}{l}\text { Range } \\
(\mathrm{msec})\end{array}$ & $n$ \\
\hline 204 & 11 & 64.8 & 59,72 & $5 / 5$ \\
& 12 & 86 & & $1 / 1$ \\
& 13 & 89.0 & 88,90 & $2 / 2$ \\
208 & 11 & 53.7 & 46,61 & $4 / 4$ \\
61 & 11 & 50.8 & 40,65 & $3 / 3$ \\
& 12 & 53.5 & 40,67 & $2 / 2$ \\
115 & 11 & 75 & & $1 / 1$ \\
& 12 & 83.9 & 61,96 & $4 / 3$ \\
\hline
\end{tabular}

Latencies were calculated from the onset of the shortening stimulus. These data are from semi-intact preparations with the stimulating electrodes implanted between segments 3 and 4 . The sample sizes are small because it was often difficult to determine the precise start of PSPs in swim circuit cells, which can show spontaneous variations in membrane potential; thus, precise latency values could not be obtained from all of the cells tested. It was uniformly clear, however, in all of the cells 204, 208, 61 , and 115 tested that the latencies were $<100 \mathrm{msec}$. For cases in which $n=1$, that value is given in the mean column. The range is given as minimum, maximum. 

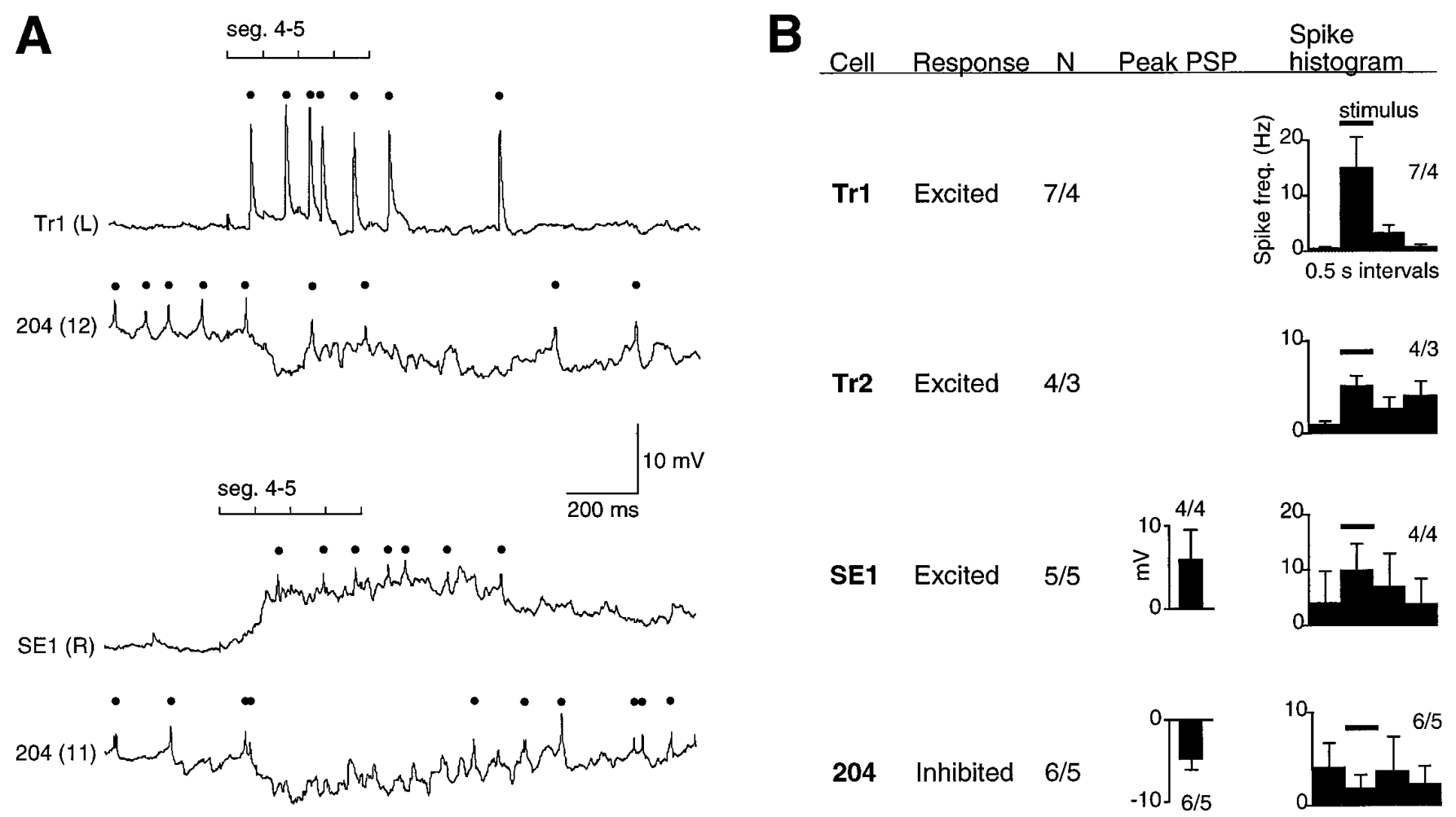

Figure 5. The responses of swim circuit neurons during shortening in reduced semi-intact preparations with the head brain exposed. $A$, Pairwise recordings demonstrating that shortening stimuli excited cells Tr1 and SE1 at the same time that they inhibited cell 204. The sets of pairwise recordings were not made concurrently, which is indicated by the distinct stimulus bars for the two sets. Dots indicate spikes. $B$, Summary of responses. Sample sizes are given as number of cells/number of preparations tested. From one to six trials were obtained from each cell. Responses were classified qualitatively by eye. Depolarizing responses accompanied by an increase in spike frequency were classified as excitatory; hyperpolarizing responses accompanied by a decrease in spike frequency were classified as inhibitory. All of the cells showed qualitatively consistent responses (i.e., excitation or inhibition) from trial to trial and from preparation to preparation. Where possible, peak PSPs and spike histograms were quantified for the responses (means were computed for each cell, from all of the trials obtained for that cell; from these were computed the overall mean + SDs for the set of cells, which are shown). Recordings from cells $\operatorname{Tr} 1$ and $\operatorname{Tr} 2$ did not show EPSPs; instead, bursts of spikes rose directly from the resting potential. Cell 204 recordings were made from segmental ganglia 10-13.

conclusion, but they at least raise the possibility that the two cells do not entirely share inputs.

The $\mathrm{S}$ cell, which is activated during shortening and makes up the fastest interneuronal pathway in the nerve cord (Shaw and Kristan, 1995a), is known to make an excitatory synapse onto cells 61 and 21 (Nusbaum and Kristan, 1986). This connection presumably accounts for at least a portion of the excitation received by cells 61 and 21 during shortening and is likely to account for the particularly short latency observed for the cell 61 response (Table 1). In support of this, in one preparation in which the $S$ cell and cell 61 were recorded simultaneously in the same ganglion, there was just a 4 msec latency from the first spike in the S cell to the start of the EPSP in cell 61 (Fig. 7). The S cell makes no connections with cell 204 (Weeks, 1982a) or cell 208 (Weeks, 1982b).

\section{DISCUSSION}

The pattern of responses shown by swim circuit interneurons during shortening is illustrated in Figure 8. Cell 204, a command-like cell at the gating level, and cell 208, an oscillator cell, receive a strong and fast inhibitory signal during shortening. Most of the other cells, including the remainder of the command-like cells and both of the highest-level cells of the circuit, are excited by stimuli that produce shortening as well as stimuli that produce swimming. These results imply that most of the command-like neurons of the swim circuit do not act as behavior-dedicated decision points for the choice between swimming and shortening. Furthermore, they imply that the decision about whether to swim or shorten does not occur at the highest level of the swim circuit, because cells at this level are unselectively activated by stimuli that cause both behaviors. Instead, the decision seems to be delegated to lower levels, in particular to cells 204 and 208.

There are some potential complications in the interpretation of our results. One argument might be that given the present results, the swim-activating neurons should not be classified as higher-level swim control cells at all. Perhaps, according to this argument, they act simply as sensory relays or filters, transmitting information from the sensory to the gating level. We find this objection unconvincing. Although it may be that the swimactivating cells are not best thought of as exclusively "swim" neurons, a point discussed below, we do believe that the available evidence supports their classification as higher-order motor control elements. First and foremost, individual depolarization of these cells can initiate swimming episodes. Their synaptic connections are consistent with this effect: both excite gating-level neurons, in particular cell 204 (Fig. 8). They also have diverse synaptic effects on other neurons (not indicated in Fig. 8). Cell Tr1 strongly excites the Retzius cells (Brodfuehrer and Friesen, 1986a), which release serotonin, a neuromodula- 


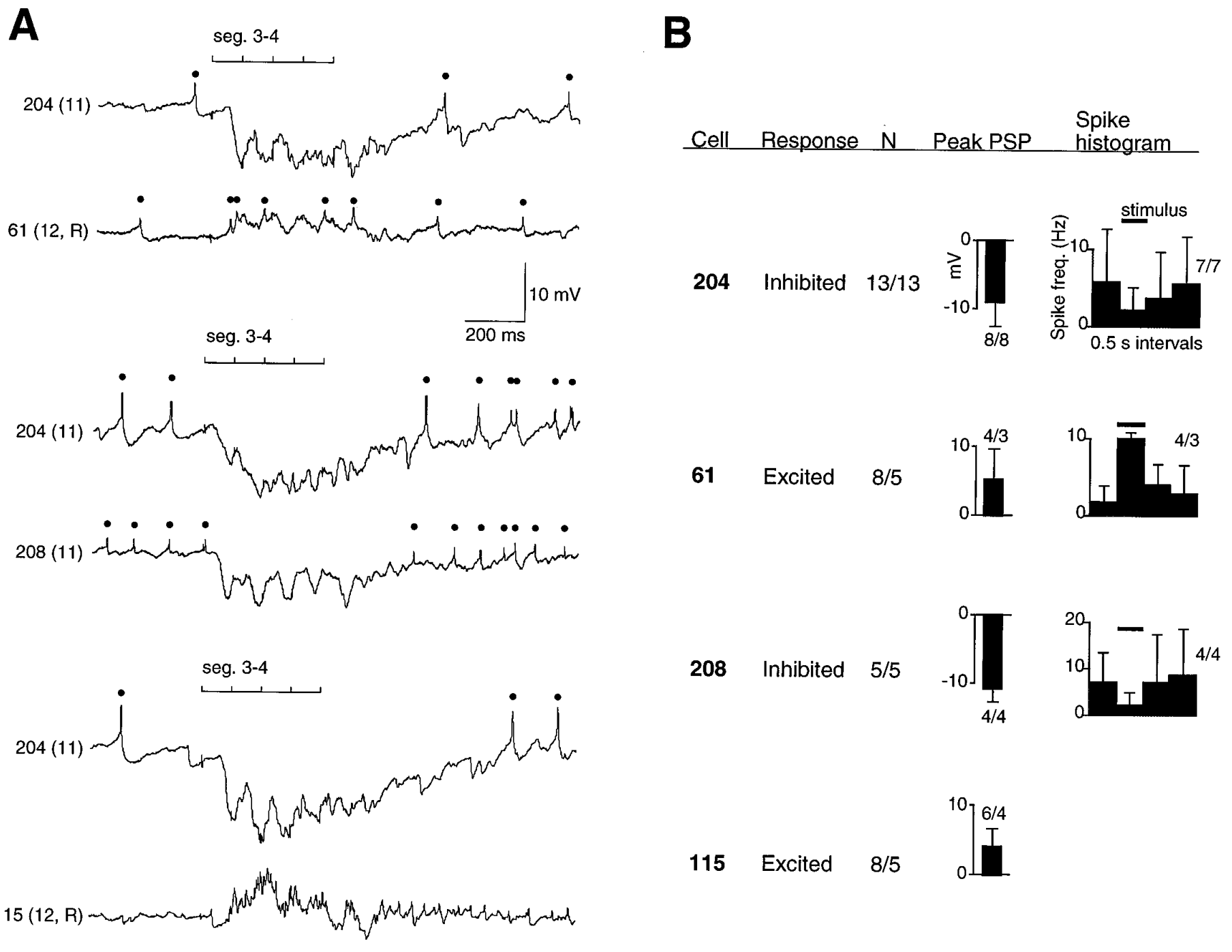

Figure 6. The responses of swim circuit neurons during shortening in semi-intact preparations. $A$, Pairwise recordings demonstrating that shortening stimuli excited cell 61, inhibited cell 208, and excited cell 115 at the same time that they inhibited cell 204. The sets of pairwise recordings were not made concurrently, which is indicated by the distinct stimulus bars. Dots indicate spikes. Spikes are not marked in the cell 115 recording because cell 115 has small somatic spikes that were difficult to distinguish individually in the midst of a compound EPSP; it was clear, however, that the EPSP caused an increase in the spike rate of the cell. $B$, Summary of responses. Data are presented as in Figure 5B. Recordings were made from segmental ganglia 11-13. From one to three trials were obtained from each cell. All of the cells showed qualitatively consistent responses (i.e., excitation or inhibition) from trial to trial and from preparation to preparation; the responses were classified as for Figure 5. A histogram is not shown for cell 115 because its small somatic spikes were difficult to count individually. It was not possible to quantify data from all of the cells tested, because recordings from some of the cells suffered from movement artifacts (see Materials and Methods); this accounts for the smaller number of cells that contributed to the computation of peak PSPs and spike histograms.

tor with multiple effects in the leech (Willard, 1981; Lent and Dickinson, 1984; Wittenberg and Kristan, 1992a). Cell SE1 powerfully excites a number of motor neurons and may play a role in regulating their activity levels (Brodfuehrer et al., 1995b). It is true, however, that cell Tr1 takes direct inputs from mechanosensory neurons (Brodfuehrer and Friesen, 1986b). To a degree, how "sensory" or "motor" these interneurons are considered to be remains a matter of interpretation. Aside from these concerns, our results for the gating and oscillator levels, taken independently, also illustrate the point that inhibition is not targeted selectively to higher-level neurons.

Although the observed pattern of responses defies easy explanation, it is possible to speculate on its rationale. Cell 204 is a particularly powerful swim initiator, more effective and reli- able than the other gating neurons (Nusbaum and Kristan, 1986). Cell 208 is a major target for excitatory inputs from the gating level and is also unique in that it is the only oscillator cell that makes excitatory connections onto other oscillator cells (Friesen, 1989a). It may be that these two neurons play an especially pivotal role in the swim circuit, such that inhibiting them is sufficient to shut down swimming. An interesting feature of the results can be seen by comparing the response pattern to the synaptic connections in Figure 8. Cells $\operatorname{Tr} 1$ and SE1, which are excited during shortening, have excitatory synapses with cell 204, which is inhibited during shortening. This means that during shortening, cells Tr1 and SE1 exert an "inappropriate" excitatory effect on cell 204 that must be overridden by other, inhibitory inputs to cell 204. An analogous situation exists between cells 61 and 21 and cell 208 . 


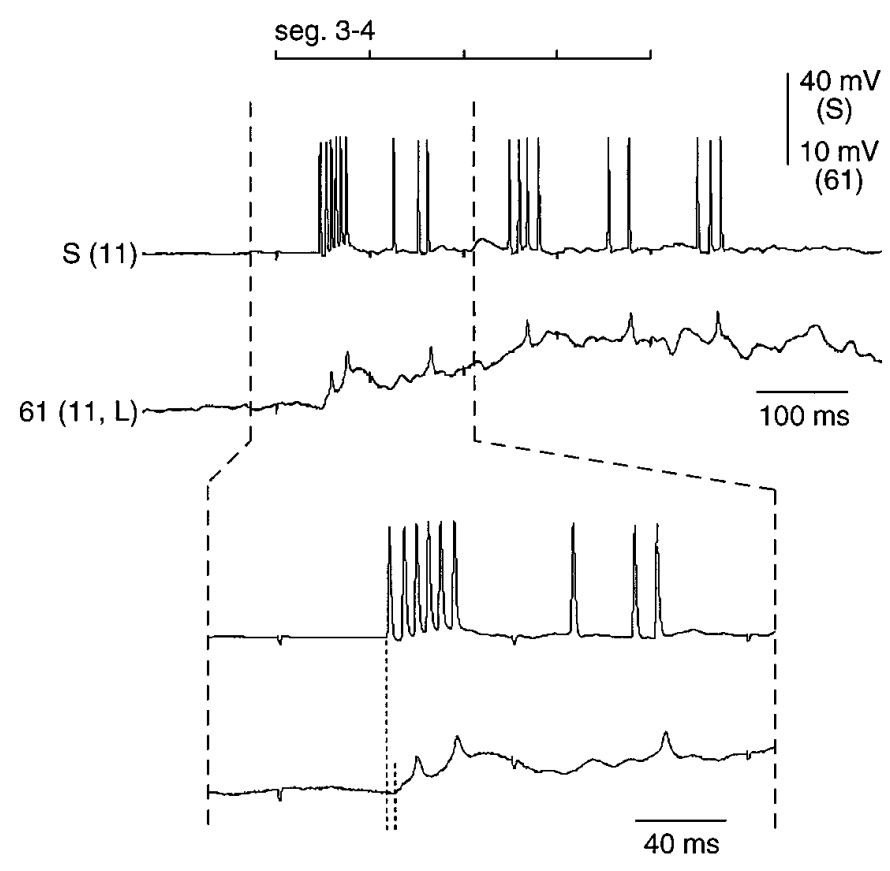

Figure 7. Pairwise recording from the $\mathrm{S}$ cell and cell 61 during shortening in a semi-intact preparation. The first spike in the $S$ cell, and the start of the EPSP in cell 61, are indicated by the dotted lines. The response latencies (from stimulus onset) are $48 \mathrm{msec}$ for the $\mathrm{S}$ cell and $52 \mathrm{msec}$ for cell 61.

\section{Dedicated versus multifunctional neurons}

The swim circuit neurons that are inhibited during shortening may be dedicated to the swimming behavior alone, whereas the cells that are activated in both behaviors are potentially multifunctional (Getting and Dekin, 1985). Cells 204 and 208 are the clearest candidates for swim-dedicated neurons and seem to play a specialized role in the decision between the behaviors. Our results add to a growing body of evidence that cell 204, in particular, acts as a decision point at which inputs that promote and suppress swimming converge (Brodfuehrer and Burns, 1995). As an aside, however, cell 204 also shows weak excitation during another locomotor behavior: crawling (Kristan et al., 1988). This may indicate that cell 204 contributes to crawling as well as swimming, or it may be related to regulating the probability of switching behaviors from crawling to swimming.

An especially perplexing feature of the results is that the highest-level neurons of the swim circuit, the swim-activating cells, are active during both behaviors. What role might these neurons normally play in behavior? We see two possibilities. One is that during shortening these cells "vote" for the animal to swim, but this effect is overridden by other neurons that vote for shortening. The other possibility is that these cells are truly multifunctional; that is, they serve a more general function than simply acting as swim activators, perhaps assisting to activate multiple behaviors, with the particular behavior to be expressed selected at lower levels. At least one attribute of cell SE1 is consistent with the second proposal: one of the motor neurons that it excites is the L cell (Brodfuehrer et al., 1995b), which is involved in shortening but not in swimming (Shaw and Kristan, 1995a). Regardless of which of these possibilities is correct, these higher-level neurons can be said to act in a "distributed" manner (Altman and Kien, 1987; Wu et al.,

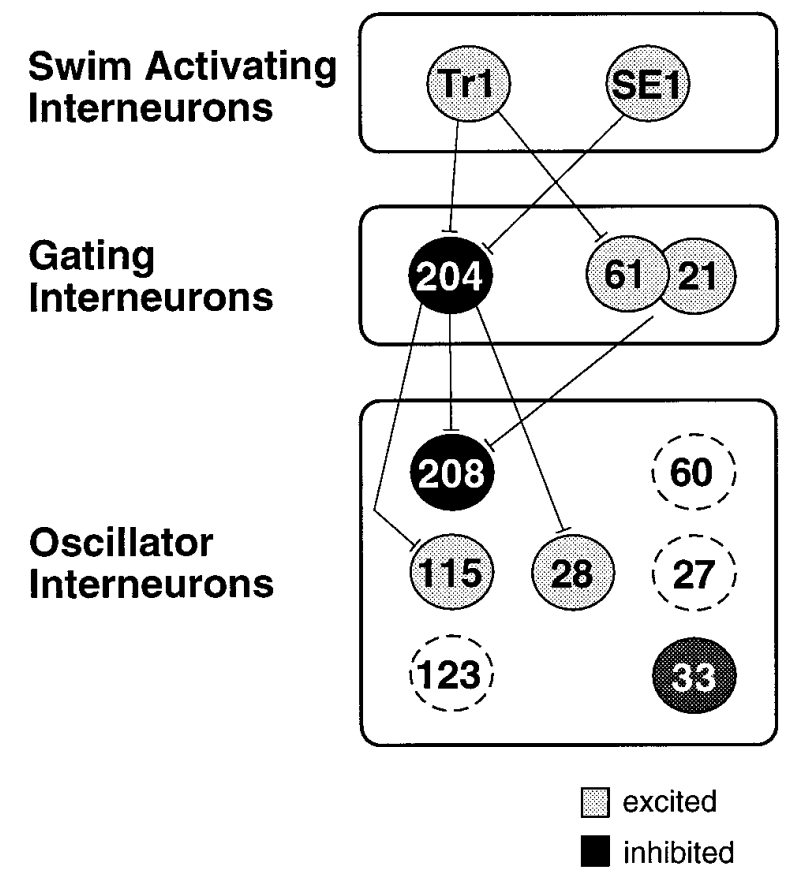

Figure 8. Schematic diagram summarizing the responses of swim circuit neurons during shortening. All of the identified swim-activating, gating, and oscillator interneurons are shown. For the sake of clarity, the only synaptic connections displayed are those that are known to link adjacent functional levels in H. medicinalis (Friesen, 1989a; Brodfuehrer et al., 1995 b); the $\perp$ symbol indicates an excitatory synapse. The feedback from the oscillator to the gating level is not represented in this diagram [with the exception of an identified synapse from cell 208 to cell 21 (Nusbaum, 1986), the synaptic basis of this feedback is not known]. Cell 33 is less darkly shaded than cells 204 and 208, because it is inhibited more weakly and with longer latency. Cells 123, 60, and 27 are shown with dotted outlines because these cells, which are difficult to locate and identify, were not tested.

1994), as part of a network in which the competing effects of a number of neurons are combined to yield an overall outcome (Lockery and Kristan, 1990). It may be too that in addition to any short-term effects, excitation of the swim-activating cells contributes to a longer-term arousal of the animal. Such arousal could be specific to swimming, increasing the probability that the leech will swim for some period of time, or it could affect multiple behaviors, increasing the probability of all of them.

Candidate multifunctional neurons lie at the gating and oscillator levels as well as at the swim-activating level. It has been shown that cells 61 and 21, which contain serotonin, can produce sensitization of the local bend reflex (Lockery and Kristan, 1991); it may be that the role of these cells has more to do with general arousal than swimming specifically. The finding that they are considerably less effective as swim initiators than cell 204 (Nusbaum and Kristan, 1986) is consistent with this idea. Cell 115 is in fact a known multifunctional neuron: it has been shown to be involved in local bending (Lockery and Kristan, 1990) and local shortening (Wittenberg and Kristan, 1992b) as well as swimming. The present results raise the possibility that it contributes to whole-body shortening as well. Ultimately, of course, the question of whether a neuron is multifunctional must be examined experimentally, by demonstrating that it makes a detectable contribution to more than one behavior. This has yet to be tested for any of the swim circuit neurons in shortening. 


\section{Roles of other head-brain neurons}

In addition to the swim-activating neurons, other head-brain cells that can affect swimming have been identified (Brodfuehrer et al., 1995a). Trigger cell 2 (Tr2) is a neuron that was classified initially as a swim-trigger cell, but recently it has been reported to be more effective at terminating swimming than initiating it (O'Gara and Friesen, 1995). Cell Tr2 is excited during shortening (Fig. $5 B$ ), but we think it unlikely that it plays an important role in the decision between the behaviors, because the rate at which it is activated during shortening is far below that required for it to terminate swimming (O'Gara and Friesen, 1995). Also, cell $\operatorname{Tr} 2$ has no direct inhibitory effects on cells 204 and 208 (Brodfuehrer and Friesen, 1986a). Another neuron that can terminate swimming, swim inhibitor 1 (SIN1), has been described recently (Brodfuehrer and Burns, 1995). We have not tested cell SIN1, but it cannot account for the fast and powerful inhibition of cells 204 and 208 seen during shortening, because its effects on these cells are indirect (204) or nonexistent (208) (Brodfuehrer and Burns, 1995).

\section{General considerations: diversity among command-like neurons}

It is natural to assume that a neuron that can initiate a given behavior is dedicated to that behavior, that its functional role should be defined primarily in terms of that behavior. This is likely to be true much of the time. Cell 204 provides an example from the present study. There are examples in other systems as well, cited in the introductory remarks. Perhaps the clearest of these cases is that of the lateral giant neuron of the crayfish, which evokes a somersaulting tailflip; selective inhibition of the lateral giant underlies the behavioral suppression of the tailflip reflex during feeding (Krasne and Lee, 1988). However, it need not always be true that command-like neurons act as behaviordedicated decision points. In the present study we found that all of the command-like neurons of the swim circuit except cell 204 were activated by stimuli that produce shortening as well as swimming. In a few other systems, also, higher-order neurons that may play roles in multiple behaviors have been identified (Ritzmann et al., 1980; Xin et al., 1996). The general lesson is that neurons that share the property of being sufficient to initiate a given behavior when artificially stimulated may serve quite different functions in the normal decision-making processes that allow an animal to choose between behaviors.

\section{REFERENCES}

Altman JS, Kien J (1987) A model for decision making in the insect nervous system. In: Nervous systems in invertebrates (Ali M, ed), pp 621-643. New York: Plenum.

Bagnoli P, Brunelli M, Magni F (1972) A fast conducting pathway in the central nervous system of the leech Hirudo medicinalis. Arch Ital Biol 110:35-51.

Brodfuehrer PD, Friesen WO (1986a) Initiation of swimming activity by trigger neurons in the leech subesophageal ganglion. I. Output connections of Tr1 and Tr2. J Comp Physiol [A] 159:489-502.

Brodfuehrer PD, Friesen WO (1986b) Initiation of swimming activity by trigger neurons in the leech subesophageal ganglion. III. Sensory inputs to Tr1 and Tr2. J Comp Physiol [A] 159:511-519.

Brodfuehrer PD, Friesen WO (1986c) Control of leech swimming activity by the cephalic ganglia. J Neurobiol 17:697-705.

Brodfuehrer PD, Burns A (1995) Neuronal factors influencing the decision to swim in the medicinal leech. Neurobiol Learn Mem 63:192-199.

Brodfuehrer PD, Debski EA, O'Gara BA, Friesen WO (1995a) Neuronal control of leech swimming. J Neurobiol 27:403-418.

Brodfuehrer PD, Parker HJ, Burns A, Berg M (1995b) Regulation of the segmental swim-generating system by a pair of identified interneurons in the leech head ganglion. J Neurophysiol 73:983-992.
Edwards DH (1991) Mutual inhibition among neural command systems as a possible mechanism for behavioral choice in crayfish. J Neurosci 11:1210-1223.

Friesen WO (1989a) Neuronal control of leech swimming movements. In: Neuronal and cellular oscillators (Jacklet JW, ed), pp 269-316. New York: Marcel Dekker.

Friesen WO (1989b) Neuronal control of leech swimming movements. II. Motor neuron feedback to oscillator cells 115 and 28. J Comp Physiol [A] 166:205-215.

Friesen WO, Poon M, Stent GS (1978) Neuronal control of swimming in the medicinal leech. IV. Identification of a network of oscillatory interneurones. J Exp Biol 75:25-43.

Getting PA, Dekin MS (1985) Tritonia swimming: a model system for integration within rhythmic motor systems. In: Model neural networks and behavior (Selverston AI, ed), pp 3-20. New York: Plenum.

Huang Z, Satterlie RA (1990) Neuronal mechanisms underlying behavioral switching in a pteropod mollusc. J Comp Physiol [A] 166:875-887.

Jing J, Gillette R (1995) Neuronal elements that mediate escape swimming and suppress feeding behavior in the predatory sea slug Pleurobranchaea. J Neurophysiol 74:1900-1910.

Kovac MP, Davis WJ (1980) Neural mechanism underlying behavioral choice in Pleurobranchaea. J Neurophysiol 43:469-487.

Krasne FB, Lee SC (1988) Response-dedicated trigger neurons as control points for behavioral actions: selective inhibition of lateral giant command neurons during feeding in crayfish. J Neurosci 8:3703-3712.

Kristan Jr WB, Stent GS, Ort CA (1974) Neuronal control of swimming in the medicinal leech. I. Dynamics of the swimming rhythm. J Comp Physiol 94:97-119.

Kristan Jr WB, McGirr SJ, Simpson GV (1982) Behavioural and mechanosensory neurone responses to skin stimulation in leeches. J Exp Biol 96:143-160.

Kristan Jr WB, Wittenberg G, Nusbaum MP, Stern-Tomlinson W (1988) Multifunctional interneurons in behavioral circuits of the medicinal leech. Experientia 44:383-389.

Kupfermann I, Weiss KR (1978) The command neuron concept. Behav Brain Sci 1:3-10.

Lent CM, Dickinson MH (1984) Serotonin integrates the feeding behavior of the medicinal leech. J Comp Physiol [A] 154:457-471.

Lockery SR, Kristan Jr WB (1990) Distributed processing of sensory information in the leech. II. Identification of interneurons contributing to the local bending reflex. J Neurosci 10:1816-1829.

Lockery SR, Kristan Jr WB (1991) Two forms of sensitization of the local bending reflex of the medicinal leech. J Comp Physiol [A] 168:165-177.

Magni F, Pellegrino M (1978) Neural mechanisms underlying the segmental and generalized cord shortening reflexes in the leech. J Comp Physiol 124:339-351.

Norekian TP, Satterlie RA (1996) Whole body withdrawal circuit and its involvement in the behavioral hierarchy of the mollusk Clione limacina. J Neurophysiol 75:529-537.

Nusbaum M (1986) Synaptic basis of swim initiation in the leech. III. Synaptic effects of serotonin-containing interneurones (cells 21 and 61) on swim CPG neurones (cells 18 and 208). J Exp Biol 122:303-321.

Nusbaum M, Kristan Jr WB (1986) Swim initiation in the leech by serotonin-containing interneurones, cells 21 and 61. J Exp Biol 122:277-302.

O'Gara BA, Friesen WO (1995) Termination of leech swimming activity by a previously identified swim trigger neuron. J Comp Physiol [A] 177:627-636.

Ort CA, Kristan Jr WB, Stent GS (1974) Neuronal control of swimming in the leech. II. Identification and connections of the motor neurons. J Comp Physiol 94:121-154.

Ritzmann RE, Tobias ML, Fourtner CR (1980) Flight activity initiated via giant interneurons of the cockroach: evidence for bifunctional trigger interneurons. Science 210:443-445.

Shaw BK, Kristan Jr WB (1993) Swim-gating neurons are inhibited during the behavioral switch from swimming to shortening in the leech. Soc Neurosci Abstr 19:348.

Shaw BK, Kristan Jr WB (1995a) The whole-body shortening reflex of the medicinal leech: motor pattern, sensory basis, and interneuronal pathways. J Comp Physiol [A] 177:667-681.

Shaw BK, Kristan Jr WB (1995b) The circuit locus of the behavioral choice between swimming and shortening in the leech. Soc Neurosci Abstr 21:1765.

Tulsi RS, Coggeshall RE (1971) Neuromuscular junctions on the muscle 
cells in the central nervous system of the leech, Hirudo medicinalis. J Comp Neurol 141:1-16.

Weeks J (1982a) Segmental specialization of a leech swim-initiating interneuron, cell 205. J Neurosci 2:972-985.

Weeks J (1982b) Synaptic basis of swim initiation in the leech. II. A pattern-generating neuron (cell 208) which mediates motor effects of swim-initiating neurons. J Comp Physiol 148:265-279.

Weeks J, Kristan Jr WB (1978) Initiation, maintenance and modulation of swimming in the medicinal leech by the activity of a single neurone. J Exp Biol 77:71-88.

Willard AL (1981) Effects of serotonin on the generation of the motor program for swimming by the medicinal leech. J Neurosci 1:936-944.
Wittenberg G, Kristan Jr WB (1992a) Analysis and modeling of the multisegmental coordination of shortening behavior in the medicinal leech. I. Motor output pattern. J Neurophysiol 68:1683-1692.

Wittenberg G, Kristan Jr WB (1992b) Analysis and modeling of the multisegmental coordination of shortening behavior in the medicinal leech. II. Role of identified interneurons. J Neurophysiol 68:1693-1707.

Wu J-Y, Cohen LB, Falk CX (1994) Neuronal activity during different behaviors in Aplysia: a distributed organization? Science 263:820-823.

Xin Y, Weiss KR, Kupfermann I (1996) A pair of identified interneurons in Aplysia that are involved in multiple behaviors are necessary and sufficient for the arterial-shortening component of a local withdrawal reflex. J Neurosci 16:4518-4528. 